\title{
Development and Validation of Polymorphic Microsatellite Loci for the NA2 Lineage of Phytophthora ramorum from Whole Genome Sequence Data
}

\author{
Marie-Claude Gagnon, Canadian Food Inspection Agency (CFIA), Ottawa, ON, Canada; Nicolas Feau and Angela L. Dale, Faculty of \\ Forestry, University of British Columbia (UBC), Vancouver, BC, Canada; Braham Dhillon, UBC and Department of Plant Pathology, Uni- \\ versity of Arkansas, Fayetteville; Richard C. Hamelin, UBC and Institut de Biologie Intégrative et des Systèmes (IBIS), Université Laval, \\ Québec, QC, Canada; Clive M. Brasier, Forest Research (FR), Farnham, SRY, United Kingdom; Niklaus J. Grünwald, Horticultural Crops \\ Research Laboratory, USDA-ARS, Corvallis, OR; and Stephan C. Brière and Guillaume J. Bilodeau, CFIA, Ottawa, ON, Canada
}

\begin{abstract}
Phytophthora ramorum is the causal agent of sudden oak death and sudden larch death, and is also responsible for causing ramorum blight on woody ornamental plants. Many microsatellite markers are available to characterize the genetic diversity and population structure of $P$. ramorum. However, only two markers are polymorphic in the NA2 lineage, which is predominant in Canadian nurseries. Microsatellite motifs were mined from whole-genome sequence data of six $P$. ramorum NA2 isolates. Of the 43 microsatellite primer pairs selected, 13 loci displayed different allele sizes among the four $P$. ramorum lineages, 10 loci displayed intralineage variation in the EU1, EU2, and/or NA1 lineages, and 12 microsatellites displayed polymorphism in the NA2 lineage. Genotyping

of 272 P. ramorum NA2 isolates collected in nurseries in British Columbia, Canada, from 2004 to 2013 revealed 12 multilocus genotypes (MLGs). One MLG was dominant when examined over time and across sampling locations, and only a few mutations separated the 12 MLGs. The NA2 population observed in Canadian nurseries also showed no signs of sexual recombination, similar to what has been observed in previous studies. The markers developed in this study can be used to assess $P$. ramorum inter- and intralineage genetic diversity and generate a better understanding of the population structure and migration patterns of this important plant pathogen, especially for the lesser-characterized NA2 lineage.
\end{abstract}

Microsatellites, also called simple-sequence repeats (SSRs) or short tandem repeats (STRs), are generally neutral molecular markers with high allelic diversity that can be amplified from small amounts of DNA. Over the last three decades, it has become increasingly common to use these markers for characterizing various aspects of model and nonmodel organisms including genetic diversity, genome mapping, parentage analysis, gene flow, and migration (Ellegren 2004; Guichoux et al. 2011; Mittal and Dubey 2009). Despite their high level of polymorphism and versatility, the use of microsatellites has often been hampered by the cost and effort needed to find and optimize a set of polymorphic loci for a species of interest (Gardner et al. 2011; Grover and Sharma 2016). In recent years, availability of nextgeneration sequencing and the development of new bioinformatics tools have simplified microsatellite marker development and increased their accessibility for species from different taxonomic groups, including fungi and oomycetes (Gagnon et al. 2016; Gardner et al. 2011; Grandjean et al. 2014; Grover and Sharma 2016; Schoebel et al. 2013).

Phytophthora ramorum Werres, De Cock \& Man in't Veld is a pathogenic oomycete responsible for causing sudden oak death (SOD) in the western United States and more recently found to be the cause of sudden larch death in the United Kingdom (Brasier and Webber 2010; Grünwald et al. 2012; Webber et al. 2010). This pathogen is also reported regularly in ornamental nurseries in North America and in Europe, where it causes ramorum blight on woody ornamental plants (Grünwald et al. 2012). P. ramorum is currently divided into four genetically divergent clonal lineages (EU1, EU2, NA1, and NA2), each named according to the continent in which it was first detected (Goss et al. 2009; Grünwald et al. 2009; Ivors et al. 2006).

Corresponding author: M.-C. Gagnon;

E-mail: marie-claude.gagnon@inspection.gc.ca

*The $\boldsymbol{e}$-Xtra logo stands for "electronic extra" and indicates that four supplementary tables and one supplementary figure are available online.

Accepted for publication 21 December 2016.

() Her Majesty the Queen in Right of Canada, as represented by the Minister of Agriculture and Agri-Food Canada
Over the years, a wide range of molecular markers have been developed to identify, describe, and distinguish $P$. ramorum lineages, including microsatellites (Goss et al. 2009; Ivors et al. 2006; Mascheretti et al. 2008; Prospero et al. 2004, 2007; Vercauteren et al. 2010, 2011b). These markers have been used to characterize the genetic diversity and migration patterns of $P$. ramorum in Europe and North America, especially for the EU1 and NA1 lineages (Croucher et al. 2013; Goss et al. 2009, 2011; Grünwald et al. 2012; Prospero et al. 2013; Vercauteren et al. 2010). However, the EU2 and NA2 lineages remain poorly characterized.

While several polymorphic microsatellite loci are available to characterize the genetic diversity of $P$. ramorum EU1 and NA1 lineages, no markers were reported as being polymorphic in the EU2 lineage (Van Poucke et al. 2012) and only two markers were reported as being polymorphic in the $P$. ramorum NA2 lineage (Prospero et al 2007; Vercauteren et al. 2011b), which is the most commonly detected in Canadian nurseries in British Columbia. Previous characterization of 44 Canadian NA2 isolates with a set of existing SSR markers, including one of the two previously reported NA2 polymorphic loci, revealed that only one multilocus genotype (MLG) was present in Canada for this lineage (Goss et al. 2011). In contrast, the same study reported 2 and 4 MLGs in Canada from only 5 NA1 and 10 EU1 isolates, respectively (Goss et al. 2011). These results raise an interesting question concerning the nature of this apparent lack of variation observed in the NA2 lineage: is this lineage intrinsically less genetically diverse than the EU1 and NA1 lineages, or is it caused by a lack of polymorphic markers specific to the NA2 lineage because of ascertainment bias (Goss et al. 2011)? To answer this question, more polymorphic microsatellite markers developed from $P$. ramorum NA2 whole genome sequence data are needed. Additionally, a new set of polymorphic markers will help better characterize the genetic diversity and population structure of the NA2 lineage in Canada.

Thus, the objectives of this study were to: (i) develop polymorphic microsatellite markers to better assess $P$. ramorum genetic diversity, primarily in the NA2 lineage, and (ii) genotype and characterize NA2 $P$. ramorum isolates collected from 2004 to 2013 from nurseries in British Columbia, Canada. To achieve these objectives, de novo assembled whole genome sequence of $P$. ramorum was used to identify putatively polymorphic microsatellite loci in the NA2 lineage and 
were tested in the four known lineages of the organism. Microsatellite markers found to be polymorphic were then used to genotype 272 Canadian and 4 American NA2 isolates to characterize the population structure and diversity of this lineage.

\section{Materials and Methods}

Whole genome sequencing, de novo assembly, and microsatellite isolation. As part of a large tree pathogen genomics project (Tree Aggressors Identification using Genomic Approaches; http:// taigaforesthealth.com/Home.aspx), the genomes of 107 P. ramorum isolates representing all four lineages (EU1, EU2, NA1, and NA2) were sequenced. Genome libraries with insert size of approximately $400 \mathrm{bp}$ were constructed on a SPRI-TE robot (Beckman Coulter Inc., Indianapolis, IN) according to the manufacturer's instructions (SPRIworks Fragment Library System I Kit, A84801). One hundred base pair (bp) from paired-end genome sequencing was done using the Illumina HiSeq 2000 (Illumina Inc., San Diego, CA) at Canada's Michael Smith Genome Sciences Centre or GSC (Vancouver, BC).

For microsatellite extraction, one isolate belonging to the NA2 lineage (04-38813; Table 1) was chosen for generation of a de novo assembly. First, the raw FASTQ reads were filtered by trimming the barcode sequence using PRINSEQ (Schmieder and Edwards 2011). Trimmed reads were assembled using ABySS version 1.3.5 (Simpson et al. 2009) at three different $\mathrm{k}-\mathrm{mer}$ values $(\mathrm{k}=44,54,64)$. The assembly at $\mathrm{k} 54$ was selected and contigs below $2 \mathrm{~kb}$ were discarded. CEGMA (Parra et al. 2007) was used to estimate the completeness of the gene space in the assembled 04-38813 isolate genome.

The Illumina raw reads for five additional NA2 isolates (Table 1) were mapped onto the assembled reference NA2 genome with the Burrows-Wheeler Aligner (BWA; Li and Durbin 2009) using the default parameters. After mapping reads from the five individual strains to the NA2 de novo assembly, the SAMtools mpileup program and the Bayesian variant calling models implemented in bcftools were used to assess variant sites ( $\mathrm{Li}$ et al. 2008). The resulting VCF file was filtered with vcftools to retain only biallelic indel variants with a coverage of at least $20 \times$. A customized Python script (ParseVCF4SSR.py; available upon request) was then used to pull out the $500 \mathrm{bp}$ region surrounding each putative microsatellite locus identified in the VCF file and was transfer to an alignment file containing FASTA formatted sequences for the NA2 de novo assembly and the five other NA2 isolates. Primers for the amplification of the putative microsatellite loci were designed from these FASTA alignments as described below.

Microsatellite primer design and optimization. Forty-three microsatellite primer pairs were designed with Primer3 web software version 4.0.0 (Koressaar and Remm 2007; Untergasser et al. 2012) using the following selection criteria: optimum (opt.) $\mathrm{T}_{\mathrm{m}}=60^{\circ} \mathrm{C}$, opt. $\mathrm{GC}$ content $=50 \%$, primer lengths of 18 to $23 \mathrm{bp}$, avoidance of secondary structures, and PCR product size between 150 and $450 \mathrm{bp}$. Loci were chosen based on their position in the genome assembly (enough space to design primers), their length and putative repeat motif ( 2 to $6 \mathrm{bp}$, perfect versus imperfect), and primer design requirements. Universal fluorescent labeling (Shimizu et al. 2002) was used to avoid the cost of genotyping with individual fluorescently labeled primers. This method comprises a universal fluorescent M13 primer (FAM or HEX-labeled) and the addition of a M13 reverse tag (5'-CAGGAAACAGCTATGACC-3') complementary to the universal fluorescent primer at the $5^{\prime}$-end of the sequence-specific forward primer (Shimizu et al. 2002). Microsatellite primer pairs were first tested on a panel of 6 to 12 isolates from all four lineages (EU1, EU2, NA1, and NA2) to assess inter- and intralineage polymorphisms (Table 2). Polymorphic loci for the NA2 lineage were then used on a panel of $272 \mathrm{Ca}-$ nadian and four American isolates of the NA2 lineage to assess variability within this lineage (Supplementary Table S1).

Genotyping. Most $P$. ramorum isolates used in this study came from nursery survey activities conducted by the Canadian Food Inspection Agency (CFIA) collection (Ottawa, ON). These isolates were collected between 2004 and 2013 from several sites in British Columbia, Canada, including retail and wholesale nurseries (Tables 1 and 2). Isolates from other countries were included as listed in Table 2. Isolates were assigned to EU1, EU2, NA1, or NA2 lineages based on sequencing, microsatellite, and/or SNP genotyping (Bilodeau et al. 2007; Van Poucke et al. 2012).

For DNA extraction, $P$. ramorum isolates were either cultured on 5\% V8 juice agar (Miller 1955) or potato dextrose agar (PDA) ( $39 \mathrm{~g} /$ /iter; Thermo Fisher Scientific) at $20^{\circ} \mathrm{C}$ under a $12 \mathrm{~h}$ photoperiod for 7 to 10 days. For isolates grown on V8, DNA was extracted from agar plugs using the DNeasy Plant Mini Kit (Qiagen Sciences, Germantown, MD), following the manufacturer's recommendations. For isolates grown on PDA, DNA was extracted with a chloroform extraction method (Möller et al. 1992) from mycelia growing on a cellophane membrane (GE Healthcare Bio-Sciences Corp., Piscataway Township, NJ) placed on top of the agar. DNA concentration was measured with Qubit assays (Life Technologies Inc., Grand Island, NY).

Amplification reactions were carried out in $25 \mu \mathrm{l}$ volumes with the following final concentrations: $1 \times$ buffer, $0.2 \mathrm{mM}$ dNTPs, $0.1 \mu \mathrm{M}$ each of M13reverse-tag, tag-forward, and reverse primers, $2 \mathrm{mM}$ $\mathrm{MgCl}_{2}, 0.04 \mathrm{U}$ Platinum Taq DNA polymerase (Life Technologies Inc., Grand Island, NY), and approximately $2 \mathrm{ng}$ of template DNA. PCR conditions were as described in Vercauteren et al. (2010): $4 \mathrm{~min}$ at $95^{\circ} \mathrm{C} ; 5$ cycles of $20 \mathrm{~s}$ at $95^{\circ} \mathrm{C}, 30 \mathrm{~s}$ at $60^{\circ} \mathrm{C}, 60 \mathrm{~s}$ at $72^{\circ} \mathrm{C} ; 20$ cycles of 'touchdown' of $20 \mathrm{~s}$ at $95^{\circ} \mathrm{C}, 30 \mathrm{~s}$ at $60^{\circ} \mathrm{C}$ to $50^{\circ} \mathrm{C}$ (dropping $0.5^{\circ} \mathrm{C}$ per cycle), $60 \mathrm{~s}$ at $72^{\circ} \mathrm{C}, 10$ cycles of $20 \mathrm{~s}$ at $95^{\circ} \mathrm{C}, 30 \mathrm{~s}$ at $50^{\circ} \mathrm{C}, 60 \mathrm{~s}$ at $72^{\circ} \mathrm{C}$; and one final step of $30 \mathrm{~min}$ at $72^{\circ} \mathrm{C}$.

Five microliters of PCR products were first run on $1.5 \%$ agarose gels to check for successful amplification and subsequently run on a 3130xl Genetic Analyzer (Life Technologies Inc., Grand Island, $\mathrm{NY)}$ to check for variability. One microliter of PCR product was suspended in $14 \mu \mathrm{l}$ of Hi-Di Formamide (Life Technologies Inc., Grand Island, NY) and $0.3 \mu$ l of GeneScan 500 LIZ dye Size Standard (Life Technologies Inc., Grand Island, NY). Two PCR products with different fluorescent dyes (FAM and HEX) were comigrated. Results were analyzed using GeneMapper v. 5 (Life Technologies Inc., Grand Island, NY).

Genetic analyses. As $P$. ramorum reproduction appears so far only asexual in nature (Grünwald et al. 2012), analysis methods suitable to address clonality in populations were chosen, as reviewed by Arnaud-Haond et al. (2007). For all loci providing clear electropherograms and displaying polymorphism (more than one allele) in the NA2 lineage, we estimated the overall number of alleles, the Simpson's

Table 1. Phytophthora ramorum NA2 isolates used for whole genome sequencing, de novo assembly, and microsatellite extraction

\begin{tabular}{|c|c|c|c|c|c|c|c|}
\hline Isolate & Year & Origin $^{a}$ & Host & Source & Total Reads & $\%$ reads mapped & Average coverage \\
\hline $04-20470$ & 2004 & BC, Canada & Rhododendron sp. & CFIA $^{\mathrm{b}}$ & $41,444,884$ & $76.2 \%$ & 55.0 \\
\hline $04-38813$ & 2004 & BC, Canada & Viburnum tinus & CFIA & $35,833,670$ & $74.4 \%$ & 49.0 \\
\hline $05-16845$ & 2005 & BC, Canada & Ardisia sp. & CFIA & $54,060,726$ & $76.5 \%$ & 79.0 \\
\hline 08-0688 & 2008 & BC, Canada & Rhododendron sp. & CFIA & $57,032,690$ & $74.8 \%$ & 79.0 \\
\hline $10-3885$ & 2010 & BC, Canada & Leucothoe fontanesiana & CFIA & $47,350,570$ & $75.1 \%$ & 66.0 \\
\hline $10-4389 a$ & 2010 & BC, Canada & Rhododendron sp. & CFIA & $48,100,884$ & $75.6 \%$ & 67.0 \\
\hline Mean & $\mathrm{N} / \mathrm{A}^{\mathrm{c}}$ & N/A & N/A & N/A & $47,303,904$ & $75.4 \%$ & 65.8 \\
\hline
\end{tabular}

${ }^{\mathrm{a}} \mathrm{BC}=$ British Columbia.

${ }^{\mathrm{b}}$ Canadian Food Inspection Agency.

${ }^{\mathrm{c}}$ Not applicable. 
index of diversity (1-D; Simpson 1949), expected heterozygosity (Nei 1978), and genotypic evenness with the E5 (Pielou 1975). We also calculated a genotype accumulation curve with 1,000 resampling without replacement to assess if the loci developed in this study were powerful enough to discriminate between the different $P$. ramorum NA2 isolates genotyped in our panel.

For all Canadian isolates genotyped, we calculated the observed number of multilocus genotypes (MLGs), the expected number of MLGs at sample size $\geq 10$ based on the rarefaction method (Hurlbert 1971; Petit et al. 1998), expected heterozygosity (Nei 1978), genotypic diversity as measured by Stoddart and Taylor's index G (Stoddart and Taylor 1988), and genotypic evenness (Pielou 1975). We also calculated the standardized index of association $\operatorname{rbar}_{d}$ (Agapow and Burt 2001) with 1,000 permutations to determine if $P$. ramorum populations were in linkage disequilibrium. These statistics were calculated for all Canadian NA2 isolates genotyped (272) and also for NA2 P. ramorum populations separated by sampling year (2004 to 2013, with the exception of 2012 where no NA2 isolates were detected). Finally, NA2 P. ramorum MLG distributions were produced per year and per nursery, for nursery sites reporting five findings or more, or less than five findings when a rare MLG was involved.

To assess the relationships among the NA2 P. ramorum MLGs, we calculated a distance matrix using Bruvo's genetic distance, which takes microsatellite repeat number into account and allows combined analysis of diploids and polyploids (Bruvo et al. 2004). A minimum spanning network was then calculated from this distance matrix. All analyses were suitable to allow combined analysis of diploids and polyploids and were conducted using RStudio (version 0.98.501 and R version 3.0.2; R Core Team, Vienna, Austria) and the package Poppr (Kamvar et al. 2014).

\section{Results}

Whole genome sequencing and de novo assembly. The $46 \mathrm{Mb}$ assembled genome for the NA2 lineage $(P$. ramorum isolate 0438813) was composed of 3,919 contigs, with the longest contig being $\sim 300 \mathrm{~kb}$ (Supplementary Table S2). For the five isolates chosen for microsatellite extraction, an average of $75 \%$ of the reads mapped to the NA2 reference genome, and the average mapping coverage was $65.8 \times$ (Table 1). Microsatellite primer pairs were designed for 43 out of the 102 variant sites identified, as previously described (see whole genome sequencing, de novo assembly, and microsatellite isolation).

Polymorphic loci observed across the four $P$. ramorum lineages. Both inter- and intralineage variation was observed among the 43 microsatellite primer pairs designed from the NA2 genome sequence data. Thirteen loci displayed different allele sizes among the four $P$. ramorum lineages (Supplementary Table S3). Ten microsatellite primer pairs displayed intralineage variation in the EU1, EU2, and/or NA1 lineages. Four of these loci displayed variation

Table 2. Phytophthora ramorum isolates used in the screening of candidate microsatellite loci polymorphic in the NA2 lineage

\begin{tabular}{|c|c|c|c|c|c|c|}
\hline Isolate & Other number & Year & Origina $^{a}$ & Host & Lineage & Source \\
\hline 03-0107 & & 2003 & BC, Canada & Rhododendron sp. & EU1 & $\mathrm{CFIA}^{\mathrm{b}}$ \\
\hline 04-17031 & & 2004 & BC, Canada & Rhododendron sp. & EU1 & CFIA \\
\hline 04-17036 & & 2004 & BC, Canada & Rhododendron sp. & EU1 & CFIA \\
\hline 08-2933 & & 2008 & BC, Canada & Pieris japonica & EU1 & CFIA \\
\hline $11-301$ & & 2011 & BC, Canada & Viburnum davidii & EU1 & CFIA \\
\hline P1559 & PR-12-057 & 2003 & SW England, UK & Rhododendron sp. & EU1 & $\mathrm{FR}^{\mathrm{c}}$ \\
\hline P1614 & PR-12-061 & 2004 & BC, Canada & Nothofagus sp. & EU1 & $\mathrm{USDA} \mathrm{ARS}^{\mathrm{d}}$ \\
\hline P1856 & PR-12-062 & 2004 & BC, Canada & Acer platanoides & EU1 & USDA ARS \\
\hline PR-09-106 & & 2009 & OR, USA & Pieris japonica & EU1 & USDA ARS \\
\hline PR-09-150 & & 2009 & OR, USA & Rhododendron sp. & EU1 & USDA ARS \\
\hline P2111 & PR-12-035 & 2007 & N. Ireland, UK & Quercus robur & EU2 & FR \\
\hline P2460 & PR-12-036 & 2010 & N. Ireland, UK & Larix kaempferi & EU2 & FR \\
\hline P2461 & PR-12-037 & 2010 & N. Ireland, UK & Larix kaempferi & EU2 & FR \\
\hline P2561 & PR-12-038 & 2011 & Scotland, UK & Larix kaempferi & EU2 & FR \\
\hline P2566 & PR-12-039 & 2011 & N. Ireland, UK & Rhododendron ponticum & EU2 & FR \\
\hline P2586 & PR-12-040 & 2011 & Scotland, UK & Larix kaempferi & EU2 & FR \\
\hline 04-002 & & 2004 & BC, Canada & Camellia sp. & NA1 & CFIA \\
\hline $04-2231$ & & 2004 & BC, Canada & Camellia japonica 'Nuccio's Gem' & NA1 & CFIA \\
\hline 04-22572 & & 2004 & BC, Canada & Rhododendron sp. & NA1 & CFIA \\
\hline 04-35009 & & 2004 & BC, Canada & Gaultheria shallon & NA1 & CFIA \\
\hline $04-22620$ & & 2004 & BC, Canada & Rhododendron sp. & NA1 & CFIA \\
\hline $10-2807 \mathrm{a}$ & & 2010 & BC, Canada & Rhododendron sp. & NA1 & CFIA \\
\hline $10-3884 a$ & & 2010 & BC, Canada & Camellia sp. & NA1 & CFIA \\
\hline $10-6342 \mathrm{a}$ & & 2010 & BC, Canada & Rhododendron sp. & NA1 & CFIA \\
\hline $11-263$ & & 2011 & BC, Canada & Rhododendron sp. & NA1 & CFIA \\
\hline $04-437$ & & 2004 & BC, Canada & Pyracantha koidzumii 'Victory' & NA2 & CFIA \\
\hline 04-20470 & & 2004 & BC, Canada & Rhododendron sp. & NA2 & CFIA \\
\hline 04-38813 & & 2004 & BC, Canada & Viburnum tinus & NA2 & CFIA \\
\hline $05-13365$ & & 2005 & BC, Canada & Magnolia sp. & NA2 & CFIA \\
\hline $05-16845$ & & 2005 & BC, Canada & Ardisia sp. & NA2 & CFIA \\
\hline $05-15042$ & & 2005 & BC, Canada & Hamamelis sp. & NA2 & CFIA \\
\hline $05-18148$ & & 2005 & BC, Canada & Rhododendron sp. & NA2 & CFIA \\
\hline $06-7402$ & & 2006 & BC, Canada & Viburnum Bodnantense "Dawn" & NA2 & CFIA \\
\hline $07-1442$ & & 2007 & BC, Canada & Rhododendron sp. & NA2 & CFIA \\
\hline 07-S1333 & & 2007 & BC, Canada & Gaultheria sp. & NA2 & CFIA \\
\hline $09-4390$ & & 2009 & BC, Canada & Camellia sp. & NA2 & CFIA \\
\hline $10-3885$ & & 2010 & BC, Canada & Leucothoe fontanesiana & NA2 & CFIA \\
\hline $10-4389 b$ & & 2010 & BC, Canada & Rhododendron sp. & NA2 & CFIA \\
\hline
\end{tabular}

${ }^{\mathrm{a}} \mathrm{BC}=$ British Columbia; SW = South West; UK = United Kingdom; OR = Oregon; USA = United States; N. Ireland = North Ireland.

b Canadian Food Inspection Agency.

${ }^{c}$ Forest Research UK.

${ }^{\mathrm{d}}$ Horticultural Crops Research Laboratory. 
in the EU1 lineage, eight loci displayed variation in the EU2 lineage, while three loci displayed variation in the NA1 lineage (Supplementary Table S4). Interestingly, polymorphism at different loci was often observed in the same one or two isolates within each lineage, despite using only 6 to 12 isolates per lineage to assess variation. For example, isolates P1856 and PR-09-150 displayed variation in the EU1 lineage, isolates P2566 and P2586 showed polymorphism in the EU2 lineage, and isolates 04-35009 and 11-263 were variable in the NA1 lineage (Table 2). Twelve loci displayed variation in the NA2 lineage (Table 3) and were used to genotype a large panel of NA2 Canadian isolates collected between 2004 and 2013 to further assess intralineage polymorphism. Finally, among all the primer pairs designed, two loci (Pram-32 and Pram-35) were variable within all four lineages (Table 3).

Genetic analyses and the assessment of MLGs. The 12 microsatellite primer pairs polymorphic in the NA2 lineage were moderately variable, displaying 2.0 to 3.0 alleles per locus (mean 2.2; Table 3 ). This moderate level of variation was also reflected in the mean 1-D value (0.473; Table 3). Despite this moderate level of variation, all loci except one displayed a high level of expected heterozygosity (mean 0.898; Table 3) and an even distribution of alleles within loci (mean 0.943; Table 3). Although the genotype accumulation curve indicated that the 12 loci developed in this study were sufficiently powerful to uncover MLG diversity, all 12 were necessary to discriminate between the different $P$. ramorum NA2 isolates included in our panel. Indeed, more than $90 \%$ of the MLGs were detected using 11 or more markers (Supplementary Fig. S1) due to the moderate level of variation displayed at each locus.

The microsatellite loci developed for the NA2 lineage detected 12 MLGs from the 272 NA2 Canadian and the four American isolates included in our panel (Table 4). Three alleles were observed at two loci in one (Pram-28) and 10 (Pram-32) of the 12 MLGs (Table 4).

Table 3. Primers used for the amplification of polymorphic microsatellite loci in Phytophthora ramorum NA2 lineage

\begin{tabular}{|c|c|c|c|c|c|c|}
\hline Name & Primer sequence $^{\mathbf{a}, \mathbf{b}}$ & Repeat motif & Nb allele ${ }^{c}$ & $1-D^{d}$ & $\mathbf{H}_{\text {exp }}{ }^{e}$ & Evenness \\
\hline Pram-06 & $\begin{array}{l}\text { F: } C A G G A A A C A G C T A T G A C C A C G T G T C C T T G C T G A G T T T G \\
\text { R: ATGAGTGGTTCGTTCGCTTG }\end{array}$ & $(\mathrm{TG})_{18}$ & 2.0 & 0.500 & 1.000 & 1.000 \\
\hline Pram-10 & $\begin{array}{l}\text { F: } C A G G A A A C A G C T A T G A C C A G C T G C A G G A T G T G G T T C A \\
\text { R: CTTGTACAGTGCCGAATCGC }\end{array}$ & $(\mathrm{CT})_{42}$ & 2.0 & 0.500 & 1.000 & 1.000 \\
\hline Pram-15 & $\begin{array}{l}\text { F: CAGGAAACAGCTATGACCCAGCAATCAGACCCACGATG } \\
\text { R: TCATCAACGTCCACACAGAC }\end{array}$ & $(\mathrm{CCG})_{6}$ & 2.0 & 0.014 & 0.029 & 0.333 \\
\hline Pram-17 & $\begin{array}{l}\text { F: } C A G G A A A C A G C T A T G A C C G G C A A C T T G G T A G C C T T C G \\
\text { R: ACTGGCACTCCCTTCTGTAC }\end{array}$ & $(\mathrm{GC})_{8}$ & 2.0 & 0.500 & 1.000 & 1.000 \\
\hline Pram-24 & $\begin{array}{l}\text { F: CAGGAAACAGCTATGACCTCACCACCGACTCCAGTTC } \\
\text { R: GACTCAGACTCACTTGCCCA }\end{array}$ & $(\mathrm{AGC})_{9}$ & 2.0 & 0.500 & 1.000 & 1.000 \\
\hline Pram-26 & $\begin{array}{l}\text { F: } C A G G A A A C A G C T A T G A C C A C C G G T A C C A A G A A G C T C T C \\
\text { R: ATCGGCTGGCTTGTAGTTCT }\end{array}$ & $(\mathrm{CAGA})_{4}$ & 2.0 & 0.500 & 1.000 & 1.000 \\
\hline Pram-28 & $\begin{array}{l}\text { F: CAGGAAACAGCTATGACCCTCCTGCTGCTCCTCCTC } \\
\text { R: CCATCGTCCGAGCTCAGC }\end{array}$ & $(\mathrm{CGAG})_{6}$ & 3.0 & 0.500 & 0.750 & 0.980 \\
\hline Pram-32 & $\begin{array}{l}\text { F: } C A G G A A A C A G C T A T G A C C T G C G G C C A A A A G T T T A C A G A \\
\text { R: TGCGTCTATTCAAGCGATTTC }\end{array}$ & $(\mathrm{CCCACA})_{4}$ & 3.0 & 0.667 & 1.000 & 1.000 \\
\hline Pram-33 & $\begin{array}{l}\text { F: } C A G G A A A C A G C T A T G A C C C C C T A C T T T G A A C A C A C C G C \\
\text { R: GTGGGATCTGCAAAAGCACA }\end{array}$ & $(\mathrm{ATA})_{6}$ & 2.0 & 0.499 & 0.998 & 0.980 \\
\hline Pram-35 & $\begin{array}{l}\text { F: } C A G G A A A C A G C T A T G A C C C T C C A G G A A G A C G A C G A T G A \\
\text { R: GTCTCCGTCGCTTTGCTTTT }\end{array}$ & $(\mathrm{GAA})_{12}$ & 2.0 & 0.500 & 1.000 & 1.000 \\
\hline Pram-41 & $\begin{array}{l}\text { F: CAGGAAACAGCTATGACCCTTGAAACGGAGCTGCAAGT } \\
\text { R: GCCTGACTCATTCGTGTGAC }\end{array}$ & $(\mathrm{GGTA})_{3}$ & 2.0 & 0.500 & 1.000 & 1.000 \\
\hline Pram-42 & $\begin{array}{l}\text { F: CAGGAAACAGCTATGACCGATTGTCTCCGTTCTGTGCG } \\
\text { R: GGCAGATCCCATTGAGCAGA }\end{array}$ & $(\mathrm{ATCGCT})_{6}$ & 2.0 & 0.500 & 1.000 & 1.000 \\
\hline Mean & $N / A^{f}$ & N/A & 2.2 & 0.473 & 0.898 & 0.943 \\
\hline
\end{tabular}

a The M13 tag-forward sequence is in italic.

${ }^{\mathrm{b}}$ Annealing temperature going from $60^{\circ} \mathrm{C}$ to $50^{\circ} \mathrm{C}$ (dropping $0.5^{\circ} \mathrm{C}$ per cycle, see Material and Methods).

${ }^{\mathrm{c}}$ Number of alleles.

${ }^{\mathrm{d}}$ Simpson's index of diversity.

e Nei's expected heterozygosity.

${ }^{\mathrm{f}} \mathrm{N} / \mathrm{A}=$ Not applicable.

Table 4. Multilocus genotypes observed in Phytophthora ramorum Canadian isolates of the NA2 lineage

\begin{tabular}{|c|c|c|c|c|c|c|c|c|c|c|c|c|c|c|c|c|c|c|c|c|c|c|c|c|c|c|}
\hline \multirow{2}{*}{$\begin{array}{l}\begin{array}{l}\text { MLG } \\
\text { profile }^{\mathbf{a}}\end{array} \\
\text { MLG1 }\end{array}$} & \multicolumn{2}{|c|}{ Pram-06 } & \multicolumn{2}{|c|}{ Pram-10 } & \multicolumn{2}{|c|}{ Pram-15 } & \multicolumn{2}{|c|}{ Pram-17 } & \multicolumn{2}{|c|}{ Pram-24 } & \multicolumn{2}{|c|}{ Pram-26 } & \multicolumn{3}{|c|}{ Pram-28 } & \multicolumn{3}{|c|}{ Pram-32 } & \multicolumn{2}{|c|}{ Pram-33 } & \multicolumn{2}{|c|}{ Pram-35 } & \multicolumn{2}{|c|}{ Pram-41 } & \multicolumn{2}{|c|}{ Pram-42 } \\
\hline & 239 & 245 & 199 & 225 & 166 & 166 & 242 & 244 & 243 & 245 & 252 & 256 & 107 & 110 & $-b$ & 239 & 252 & 261 & 240 & 247 & 147 & 150 & 222 & 229 & 220 & 227 \\
\hline MLG2 & 239 & 245 & 199 & 225 & 164 & $164^{\mathrm{c}}$ & 242 & 242 & 243 & 243 & 256 & 256 & 107 & 107 & & 252 & 252 & - & 240 & 240 & 147 & 147 & 222 & 229 & 220 & 227 \\
\hline MLG3 & 239 & 245 & 199 & 225 & 166 & 166 & 242 & 244 & 243 & 245 & 252 & 256 & 107 & 107 & - & 239 & 252 & 261 & 240 & 240 & 147 & 150 & 222 & 229 & 220 & 227 \\
\hline MLG4 & 239 & 245 & 199 & 225 & 164 & 164 & 242 & 242 & 243 & 243 & 256 & 256 & 107 & 110 & - & 252 & 252 & - & 240 & 247 & 147 & 147 & 222 & 229 & 220 & 227 \\
\hline MLG5 & 239 & 245 & 199 & 225 & 166 & 166 & 242 & 244 & 243 & 245 & 252 & 256 & 107 & 107 & & 239 & 252 & $2 \overline{6} 1$ & 240 & 247 & 147 & 150 & 222 & 229 & 220 & 227 \\
\hline MLG6 & 245 & 245 & 199 & 225 & 166 & 166 & 242 & 244 & 243 & 245 & 252 & 256 & 107 & 110 & - & 239 & 252 & 261 & 240 & 247 & 147 & 150 & 222 & 229 & 227 & 227 \\
\hline MLG7 & 239 & 245 & 199 & 225 & 166 & 166 & 242 & 244 & 243 & 245 & 252 & 256 & 107 & 110 & - & 239 & 252 & 261 & 240 & 240 & 147 & 150 & 222 & 229 & 220 & 227 \\
\hline MLG8 & 239 & 245 & 199 & 225 & 166 & 166 & 242 & 244 & 243 & 243 & 252 & 256 & 107 & 110 & - & 239 & 252 & 261 & 240 & 247 & 147 & 150 & 222 & 229 & 220 & 227 \\
\hline MLG9 & 239 & 245 & 199 & 225 & 166 & 166 & 242 & 244 & 243 & 245 & 252 & 256 & 95 & 107 & 110 & 239 & 252 & 261 & 240 & 247 & 147 & 150 & 222 & 229 & 220 & 227 \\
\hline MLG10 & 239 & 245 & 199 & 225 & 166 & 166 & 242 & 244 & 243 & 245 & 252 & 256 & 107 & 110 & - & 239 & 252 & 261 & 247 & 247 & 147 & 150 & 222 & 229 & 220 & 227 \\
\hline MLG11 & 245 & 245 & 225 & 225 & 166 & 166 & 242 & 244 & 243 & 245 & 252 & 256 & 107 & 107 & - & 239 & 252 & 261 & 240 & 240 & 147 & 150 & 222 & 222 & 227 & 227 \\
\hline MLG12 & 239 & 239 & 199 & 199 & 166 & 166 & 242 & 244 & 243 & 245 & 252 & 256 & 107 & 110 & _ & 239 & 252 & 261 & 240 & 247 & 147 & 150 & 229 & 229 & 220 & 220 \\
\hline
\end{tabular}

\footnotetext{
a Multilocus genotype profile.

b No allele or missing data.

${ }^{c}$ Alleles in bold differ from alleles observed in MLG1.
} 
From 2004 to 2013, the P. ramorum NA2 Canadian population was dominated by MLG1, which represented $90 \%$ of all isolates (Table 5). This genotype is situated at the center of the minimum spanning network built using Bruvo's genetic distance (Fig. 1). The four American NA2 isolates genotyped also belonged to MLG1. The other genotypes, detected more than once and over multiple years, were MLG3 (2.57\%), MLG5 (2.57\%), and MLG7 (1.47\%). These genotypes are also located near the center of the network (Fig. 1). The last eight MLGs observed in our panel were detected once or twice during a single year and were mostly situated at the margins of the minimum spanning network (Table 5 and Fig. 1).
Temporal and spatial distribution of the $P$. ramorum NA2 lineage MLGs. Overall, the highest number of NA2 P. ramorum isolates recovered from nurseries in British Columbia, Canada, were detected in 2007 with 91 records. This was also the year where the highest numbers of MLGs were observed (Table 6). However, when the rarefaction method was used, the years with the highest number of expected MLGs were 2004 and 2005. These two years were also among those with the highest genotypic diversity (Stoddard and Taylor $\mathrm{G}$ index; Table 6), the highest expected heterozygosity, and the highest genotypic evenness $\left(\mathrm{E}_{5}\right.$; Table 6$)$, despite the moderate levels of detections that occurred during those years.

Table 5. Isolate distribution per year for each multilocus genotype (MLG) of Phytophthora ramorum NA2 lineage detected between 2004 and 2013 in British Columbia, Canada

\begin{tabular}{|c|c|c|c|c|c|c|c|c|c|c|c|}
\hline \multirow[b]{2}{*}{ MLG profile } & \multicolumn{2}{|c|}{ Isolates } & \multicolumn{9}{|c|}{ Year } \\
\hline & Number & $\%$ of total & 2004 & 2005 & 2006 & 2007 & 2008 & 2009 & 2010 & 2011 & 2013 \\
\hline MLG1 & 245 & 90.07 & 12 & 19 & 74 & 81 & 36 & 7 & 3 & 6 & 7 \\
\hline MLG2 & 1 & 0.37 & 1 & - & - & - & - & - & - & - & _- \\
\hline MLG3 & 7 & 2.57 & 1 & 1 & 1 & 4 & - & - & - & - & - \\
\hline MLG4 & 1 & 0.37 & 1 & - & - & - & - & - & - & - & - \\
\hline MLG5 & 7 & 2.57 & _ & $-\overline{2}$ & 2 & 1 & 2 & - & - & - & - \\
\hline MLG6 & 1 & 0.37 & $\begin{array}{l}- \\
-\end{array}$ & 1 & - & - & - & - & - & - & $\begin{array}{l}- \\
-\end{array}$ \\
\hline MLG7 & 4 & 1.47 & - & - & 1 & 3 & _- & - & - & - & - \\
\hline MLG8 & 1 & 0.37 & - & - & _ & 1 & - & - & _- & _- & _- \\
\hline MLG9 & 1 & 0.37 & _- & _- & - & 1 & - & - & - & - & - \\
\hline MLG10 & 2 & 0.73 & - & _- & - & _- & 2 & - & - & - & - \\
\hline MLG11 & 1 & 0.37 & - & - & - & - & 1 & - & - & - & - \\
\hline MLG12 & 1 & 0.37 & - & - & - & - & _- & - & $\overline{1}$ & - & - \\
\hline Total & 272 & 100.00 & $\overline{15}$ & $\overline{23}$ & $\overline{78}$ & $\overline{91}$ & $\overline{41}$ & $\overline{7}$ & 4 & $\overline{6}$ & $\overline{7}$ \\
\hline
\end{tabular}

\section{POPULATION}
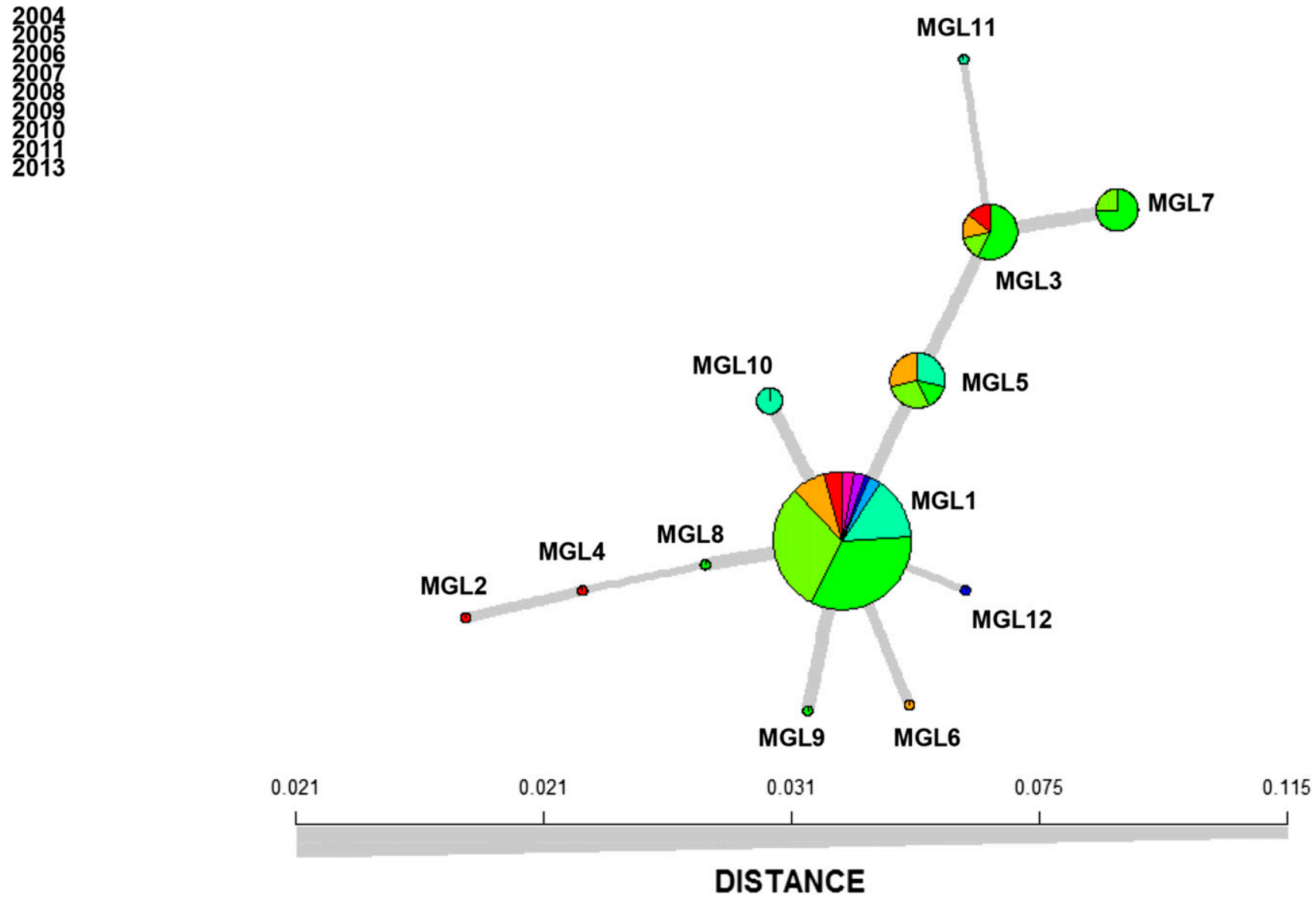

Fig. 1. Minimum spanning network of the 12 multilocus genotypes (MLGs) observed in the NA2 clonal lineage of Phytophthora ramorum over 10 years in Canada. 
From 2004 to 2013,17 out of 32 nurseries had five or more findings of NA2 P. ramorum, or less than five findings but a rare MLG. The most common genotype (MLG1) was found in all nurseries except one, often over multiple collection years (Table 7). The other most common genotypes (MLG3, MLG5, and MLG7) were reported from six, five, and three different nurseries, respectively (Table 7). Nursery A was responsible for the most $P$. ramorum NA2 detections, with 82 detections over 5 years, and this nursery was also host to the highest $P$. ramorum NA2 genotypic diversity, with six MLGs detected during the collection period (Table 7). Four other nurseries (G, I, O, and Q) reported around 20 detections and between 2 and 4 MLGs during the collection years (Table 7).

Finally, no signs of sexual recombination were observed in the NA2 $P$. ramorum Canadian population based on the following observations. First, a single genotype (MLG1) dominated this population from 2004 to 2013. Second, expected levels of heterozygosity were very high (mean 0.898) at all loci except one (Table 3 ) and in all the collection years (Table 6), suggesting a high rate of fixed heterozygotes. Third, the standardized index of association $\left(\operatorname{rbar}_{\mathrm{D}}\right)$ calculated for each collection year and overall NA2 Canadian $P$. ramorum isolates had $P$ values $\leq 0.002$, indicating significant linkage disequilibrium among microsatellite loci in NA2 $P$. ramorum Canadian population over all the years sampled (Table 6).

\section{Discussion}

The whole genome sequence of a $P$. ramorum isolate belonging to the NA2 lineage was used to rapidly identify and develop polymorphic microsatellite loci in this lineage. This is relevant since ascertainment bias likely reduced the usefulness of previous microsatellite markers that were developed mainly from two (EU1 and NA1) of the other three lineages of this pathogen. The level of polymorphism was sufficient to identify several MLGs and will therefore be useful for pathogen monitoring and molecular epidemiology. Other loci displaying interlineage variation and/or variation within the EU1, EU2, and NA1 lineages were also discovered while screening the markers. The loci polymorphic in the NA2 lineage allowed for the genotyping of a large panel of $P$. ramorum NA2 isolates collected in British Columbia, Canada, from 2004 to 2013. Results indicated that the Canadian $P$. ramorum NA2 population is composed of 12 MLGs, with one MLG dominant over the years and across sampling locations tested in this study, and that this population shows no sign of sexual recombination, similar to what was observed in other $P$. ramorum populations and lineages (Prospero et al. 2007; Vercauteren et al. 2010).

From the 43 microsatellite primer pairs designed from the NA2 genome sequence data, 13 displayed interlineage variation among the four $P$. ramorum lineages (EU1, EU2, NA1, and NA2). This suggests that one or more of these loci could be optimized for

Table 6. Genotypic diversity statistics for Canadian Phytophthora ramorum NA2 population from 2004 to 2013

\begin{tabular}{|c|c|c|c|c|c|c|c|c|}
\hline Year & $\mathbf{N}$ & MLG $^{\mathbf{a}}$ & eMLGb & $\mathrm{SE}^{\mathrm{c}}$ & $\mathbf{G}^{\mathbf{d}}$ & $\operatorname{Hexp}^{\mathrm{e}}$ & $E_{5}{ }^{f}$ & $\operatorname{rbar}_{D}{ }^{g}$ \\
\hline 2004 & 15 & 4 & 3.14 & 0.720 & 1.58 & 0.396 & 0.515 & $0.747^{*}$ \\
\hline 2005 & 23 & 4 & 2.56 & 0.795 & 1.44 & 0.320 & 0.489 & $0.189^{*}$ \\
\hline 2006 & 78 & 4 & 1.50 & 0.627 & 1.11 & 0.100 & 0.377 & $0.373^{*}$ \\
\hline 2007 & 91 & 6 & 2.00 & 0.825 & 1.26 & 0.207 & 0.394 & $0.286^{*}$ \\
\hline 2008 & 41 & 4 & 2.11 & 0.791 & 1.29 & 0.229 & 0.445 & $0.642 *$ \\
\hline 2009 & 7 & 1 & 1.00 & 0.000 & 1.00 & 0.000 & $\mathrm{~N} / \mathrm{A}^{\mathrm{h}}$ & N/A \\
\hline 2010 & 4 & 2 & 2.00 & 0.000 & 1.60 & 0.500 & 0.795 & $1.000 *$ \\
\hline 2011 & 6 & 1 & 1.00 & 0.000 & 1.00 & 0.000 & N/A & N/A \\
\hline 2013 & 7 & 1 & 1.00 & 0.000 & 1.00 & 0.000 & N/A & N/A \\
\hline Total & 272 & 12 & 1.94 & 0.855 & 1.23 & 0.188 & 0.333 & $0.310 *$ \\
\hline
\end{tabular}

a The number of multilocus genotypes observed.

b The number of multilocus genotypes expected based on rarefaction (for sample size $\geq 10$ ).

c Standard error based on the expected multilocus genotypes.

d Stoddart and Taylor's index of genotypic diversity.

e Nei's expected heterozygosity.

${ }^{\mathrm{f}}$ Genotypic evenness. * $=P$ value $\leq 0.002$

${ }^{\mathrm{g}}$ Standardized index of association.

${ }^{\text {h }}$ Not applicable. rapid identification of all $P$. ramorum lineages. As only 6 to 12 isolates of each lineage were tested with these loci, further optimization would be needed to find the combination of markers offering the best interlineage discrimination. Other molecular tools such as PCRRFLP (Elliott et al. 2009; Van Poucke et al. 2012) and real-time PCR assays (Bilodeau et al. 2007; Gagnon et al. 2014) exist for the identification of all four $P$. ramorum lineages. However, their use requires restriction enzymes or real-time PCR technology, decreasing their accessibility for some laboratories or increasing turnover time compared with microsatellite markers.

The screening of candidate microsatellite markers polymorphic for $P$. ramorum NA2 lineage also revealed 10 loci displaying intralineage variation in the EU1, EU2, and NA1 lineages. While several polymorphic microsatellite loci have been developed in the last decade for the EU1 and NA1 lineages (Ivors et al. 2006; Prospero et al. 2004, 2007; Vercauteren et al. 2010, 2011b), the findings presented here are the first report of microsatellite markers polymorphic for the EU2 lineage, together with EU1 responsible for causing sudden larch death in the United Kingdom (Brasier and Webber 2010; Van Poucke et al. 2012; Webber et al. 2010). The eight loci displaying variation in the EU2 lineage were tested on only six isolates, suggesting that these markers have good potential to reveal more polymorphism if they are used to screen a larger panel of EU2

Table 7. Genetic diversity of Canadian Phytophthora ramorum NA2 isolates partitioned by nursery and collection year. Reported are nurseries with five findings or more, or less than five findings but a rare multilocus genotype (MLG)

\begin{tabular}{|c|c|c|c|}
\hline Nursery code & Total & Year & MLG profile ${ }^{a}$ \\
\hline \multirow[t]{5}{*}{ A } & 82 & 2004 & $\mathbf{1}(1) ; \mathbf{2}(1)$ \\
\hline & & 2005 & $\mathbf{1}(16) ; \mathbf{5}(2) ; \mathbf{6}(1)$ \\
\hline & & 2006 & $\mathbf{1}(48) ; \mathbf{3}(1) ; \mathbf{5}(1) ; \mathbf{7}(1)$ \\
\hline & & 2007 & $1(3)$ \\
\hline & & 2013 & 1(7) \\
\hline \multirow[t]{2}{*}{ B } & 5 & 2004 & 1(3) \\
\hline & & 2007 & $1(2)$ \\
\hline \multirow[t]{3}{*}{$\mathrm{C}$} & 8 & 2004 & $\mathbf{1}(1)$ \\
\hline & & 2007 & 1(4) \\
\hline & & 2008 & $\mathbf{1}(3)$ \\
\hline \multirow[t]{2}{*}{$\mathrm{D}$} & 6 & 2004 & $\mathbf{1}(3) ; \mathbf{3}(1)$ \\
\hline & & 2006 & $\mathbf{1}(2)$ \\
\hline \multirow[t]{3}{*}{$\mathrm{E}$} & 8 & 2004 & $\mathbf{1}(3) ; \mathbf{4}(1)$ \\
\hline & & 2006 & $\mathbf{1}(1)$ \\
\hline & & 2007 & $\mathbf{1}(2) ; \mathbf{7}(1)$ \\
\hline $\mathrm{F}$ & 1 & 2005 & $3(1)$ \\
\hline \multirow[t]{3}{*}{ G } & 20 & 2005 & $\mathbf{1}(1)$ \\
\hline & & 2007 & $\mathbf{1}(16) ; \mathbf{3}(2)$ \\
\hline & & 2008 & $\mathbf{1}(1)$ \\
\hline \multirow[t]{3}{*}{$\mathrm{H}$} & 11 & 2005 & $\mathbf{1}(1)$ \\
\hline & & 2006 & $\mathbf{1}(7) ; \mathbf{5}(1)$ \\
\hline & & 2007 & $\mathbf{1}(2)$ \\
\hline \multirow[t]{2}{*}{ I } & 22 & 2006 & $\mathbf{1}(7)$ \\
\hline & & 2007 & $\mathbf{1}(13) ; \mathbf{3}(1) ; \mathbf{9}(1)$ \\
\hline $\mathrm{J}$ & 5 & 2007 & $\mathbf{1}(4) ; \mathbf{5}(1)$ \\
\hline K & 4 & 2007 & $\mathbf{1}(3) ; \mathbf{3}(1)$ \\
\hline $\mathrm{L}$ & 9 & 2007 & $\mathbf{1}(8) ; 8(1)$ \\
\hline \multirow[t]{2}{*}{ M } & 4 & 2007 & $\mathbf{1}(2)$ \\
\hline & & 2008 & $\mathbf{1}(1) ; \mathbf{5}(1)$ \\
\hline $\mathrm{N}$ & 7 & 2007 & $1(5) ; 7(2)$ \\
\hline \multirow[t]{3}{*}{$\mathrm{O}$} & 23 & 2008 & $\mathbf{1}(16) ; \mathbf{5}(1) ; \mathbf{1 0}(1) ; \mathbf{1 1}(1)$ \\
\hline & & 2009 & $1(3)$ \\
\hline & & 2010 & $\mathbf{1}(1)$ \\
\hline $\mathrm{P}$ & 5 & 2008 & $\mathbf{1}(5)$ \\
\hline \multirow[t]{4}{*}{ Q } & 19 & 2008 & $1(7) ; 10(1)$ \\
\hline & & 2009 & $\mathbf{1}(3)$ \\
\hline & & 2010 & $\mathbf{1}(1) ; \mathbf{1 2}(1)$ \\
\hline & & 2011 & $\mathbf{1}(6)$ \\
\hline
\end{tabular}

${ }^{a}$ Multilocus genotype (MLG) profiles for each nursery and each collection year. Numbers in bold refer to MLG number. Numbers in brackets refer to the number of isolates. 
isolates. Even though draft genomes have been published recently for several EU2 isolates from Scotland and Northern Ireland (Mata Saez et al. 2015; Sambles et al. 2015), enabling a better comprehension of this new lineage and the discovery of lineage-specific polymorphisms such as SNPs, microsatellites are still a leading tool of choice to unravel population structure and to use in routine diagnostics (Cooke and Lees 2004; Goss 2015).

Twelve of the microsatellites developed from whole genome sequence data of six NA2 isolates displayed polymorphism in the NA2 lineage. This represents a sixfold increase over the two polymorphic markers previously available for this lineage (Prospero et al. 2007; Vercauteren et al. 2011b). These new microsatellites revealed that 12 MLGs were present in a large panel of 272 P. ramorum NA2 isolates collected in British Columbia, Canada, over almost 10 years of SOD surveys conducted by the CFIA. This also represents a five- to sixfold increase over the MLG variation previously reported for the NA2 lineage in Canada and United States (Goss et al. 2009, 2011). Two of these new microsatellites show the presence of three alleles in one (Pram-28) and 10 (Pram-32) of the 12 MLGs. The presence of more than two alleles at these loci could result from the amplification of more than one locus by each primer pair, as observed for other microsatellites markers previously developed for $P$. ramorum (Vercauteren et al. 2010, 2011b). The three alleles observed at these two loci could also result from trisomy for certain MLGs at those markers. Non-Mendelian inheritance and genome instability has been observed in the single-oospore progeny of A1 $\times$ A2 experimental crosses of $P$. ramorum (Vercauteren et al. 2011a). Host-induced aneuploidy, including trisomy, has also been documented in certain NA1 and EU1 isolates of $P$. ramorum from oaks in California (Kasuga et al. 2016).

Despite the increase over the MLG variation previously reported for the NA2 lineage in Canada, the level of variation observed within this lineage is still low compared with the variation present in the EU1 and the NA1 lineages in Europe, the United States, and Canada (Croucher et al. 2013; Goss et al. 2009, 2011; Grünwald et al. 2012; Ivors et al. 2004, 2006; Prospero et al. 2007, 2013; Vercauteren et al. 2010). For example, 53 MLGs have been reported in an analysis of 228 isolates of the NA1 lineage collected from American nurseries between 2004 and 2007 using eight microsatellite loci (Goss et al. 2009). However, NA1 is an older lineage that had more time to undergo clonal divergence by accumulating novel mutations at SSR loci over space and time since being introduced to California in the 1990s.

It was previously suggested that the reduced level of variation observed for the NA2 lineage might result either from the low number of polymorphic markers available to characterize this lineage, or from intrinsic low genetic diversity present within the lineage (Goss et al. 2011). In light of the low genetic diversity revealed despite using a five/sixfold higher number of polymorphic markers to genotype the large panel of NA2 Canadian isolates, the results of this study suggest that there may indeed be a low intrinsic genetic diversity within the NA2 lineage per se. The observations that one MLG is dominant in the Canadian NA2 population and that the 12 MLGs are separated by only a few mutations both support this finding and suggest that a bottleneck may have occurred during the original NA2 introduction in British Columbia, Canada, or Washington State, U.S.A. (Goss et al. 2011; Grünwald et al. 2012). This is also congruent with the population being smaller, established over a smaller spatial range, more recent, and resulting from the effect of ongoing eradication programs. Going forward, we would expect NA2 populations to diverge clonally as has been observed for NA1 if not completely controlled by eradication.

Genotyping of 272 P. ramorum NA2 isolates indicated that 2007 was the year with the highest number of MLGs found in Canadian nurseries. However, once rarefaction was used, the highest numbers of expected MLGs were found in 2004 and 2005. These years were also among those displaying the highest levels of genetic diversity across all the samples analyzed, which is consistent with the original detection of the NA2 lineage in British Columbia, Canada, and Washington State, U.S.A., in 2004 (Goss et al. 2011; Grünwald et al. 2012). Following the first detections of Canadian NA2 isolates in 2004, sampling by CFIA inspectors increased in British Columbia nurseries, resulting in more rapid identification and eradication of infested plant material, thus decreasing the effective population size and genetic diversity of the $P$. ramorum NA2 lineage.

Only two NA2 P. ramorum MLGs (1 and 5) were detected in the same nursery (Nursery A) over different collection years. This suggests that the CFIA's eradication program (CFIA Inspection Procedure PI-010) is largely successful in stopping the spread of the $P$. ramorum NA2 lineage through the nursery pathway. However, some MLGs $(3,5,7$, and 10) have been detected in different nurseries on different years. This turnover of genotypes could result from new introductions of genotypes in nurseries every year. However, a small amount of $P$. ramorum NA2 inoculum may also remain in symptomless plants, in decomposing infected material, in the soil or in a water source after the destruction of contaminated plants, resulting in the reappearance of some genotypes over the years and in different nurseries (Swain and Garbelotto 2016; Vercauteren et al. 2013; Werres et al. 2007).

In this study, whole genome sequence data from six isolates of the $P$. ramorum NA2 lineage were used to develop microsatellite markers displaying interlineage variation and/or variation within the EU1, EU2, NA1, and NA2 lineages. The NA2 polymorphic markers were used to assess the genetic structure of the Canadian NA2 population collected from 2004 to 2013 in British Columbia. This study also represents the first report of markers polymorphic for the EU2 lineage. The addition of these new markers to the $P$. ramorum genomic toolbox will allow better characterization of this important pathogen, and will improve management of sudden oak death and ramorum blight in the years to come.

\section{Acknowledgments}

The authors would like to thank M. Newton, D. Shearlaw, A. Brar, and R. Vandenbeek (Pathogen Identification Research Lab, CFIA, Ottawa, Canada) for technical help and E. Hopwood (Genotyping/Botany Lab, CFIA, Ottawa, Canada), D. Ogunremi (Science Services, CFIA, Ottawa, Canada), and C. Dollard (Plant Research \& Strategies, CFIA, Ottawa, Canada) for editing and reviewing the manuscript. Support was provided by Genome Canada and Genome BC through the 2010 Large-Scale Applied Research Project for the TAIGA project (Tree Aggressors Identification using Genomic Approaches; http://taigaforesthealth.com/Home aspx) and CFIA Research Partnership Strategy (RPS) fund for the project OLF-P1302 .

\section{Literature Cited}

Agapow, P.-M., and Burt, A. 2001. Indices of multilocus linkage disequilibrium. Mol. Ecol. Notes 1:101-102.

Arnaud-Haond, S., Duarte, C. M., Alberto, F., and Serrao, E. A. 2007 Standardizing methods to address clonality in population studies. Mol. Ecol. 16:5115-5139.

Bilodeau, G. J., Lévesque, C. A., de Cock, A. W. A. M., Brière, S. C., and Hamelin, R. C. 2007. Differentiation of European and North American genotypes of Phytophthora ramorum by real-time polymerase chain reaction primer extension. Can. J. Plant Pathol. 29:408-420.

Brasier, C. M., and Webber, J. 2010. Plant pathology: Sudden larch death. Nature 466:824-825.

Bruvo, R., Michiels, N. K., D'Souza, T. G., and Schulenburg, H. 2004. A simple method for the calculation of microsatellite genotype distances irrespective of ploidy level. Mol. Ecol. 13:2101-2106.

Cooke, D. E. L., and Lees, A. K. 2004. Markers, old and new, for examining Phytophthora infestans diversity. Plant Pathol. 53:692-704.

Croucher, P. J. P., Mascheretti, S., and Garbelotto, M. 2013. Combining field epidemiological information and genetic data to comprehensively reconstruct the invasion history and the microevolution of the sudden oak death agent Phytophthora ramorum (Stramenopila: Oomycetes) in California. Biol. Invasions 15:2281-2297.

Ellegren, H. 2004. Microsatellites: simple sequences with complex evolution. Nat Rev. Genet. 5:435-445.

Elliott, M., Sumampong, G., Varga, A., Shamoun, S. F., James, D., Masri, S., Brière, S. C., and Grünwald, N. J. 2009. PCR-RFLP markers identify three lineages of the North American and European populations of Phytophthora ramorum. For. Pathol. 39:266-278.

Gagnon, M.-C., Bergeron, M.-J., Hamelin, R. C., Grünwald, N. J., and Bilodeau, G. J. 2014. Real-time PCR assay to distinguish Phytophthora ramorum lineages using the cellulose binding elicitor lectin (CBEL) locus. Can. J. Plant Pathol. 36:367-376.

Gagnon, M.-C., van der Lee, T. A. J., Bonants, P. J. M., Smith, D. S., Li, X., Lévesque, C. A., and Bilodeau, G. J. 2016. Development of polymorphic microsatellite loci for potato wart from next-generation sequence data. Phytopathology 106 636-644. 
Gardner, M. G., Fitch, A. J., Bertozzi, T., and Lowe, A. J. 2011. Rise of the machines-recommendations for ecologists when using next generation sequencing for microsatellite development. Mol. Ecol. Resour. 11:1093-1101.

Goss, E. M. 2015. Genome-enabled analysis of plant-pathogen migration. Annu. Rev. Phytopathol. 53:121-135.

Goss, E. M., Larsen, M., Chastagner, G. A., Givens, D. R., and Grünwald, N. J. 2009. Population genetic analysis infers migration pathways of Phytophthora ramorum in US nurseries. PLoS Pathog. 5:e1000583.

Goss, E. M., Larsen, M., Vercauteren, A., Werres, S., Heungens, K., and Grünwald, N. J. 2011. Phytophthora ramorum in Canada: Evidence for migration within North America and from Europe. Phytopathology 101:166-171.

Grandjean, F., Vrålstad, T., Diéguez-Uribeondo, J., Jelić, M., Mangombi, J., Delaunay, C., Filipová, L., Rezinciuc, S., Kozubíková-Balcarová, E., and Guyonnet, D. 2014. Microsatellite markers for direct genotyping of the crayfish plague pathogen Aphanomyces astaci (Oomycetes) from infected host tissues. Vet. Microbiol. 170:317-324.

Grover, A., and Sharma, P. C. 2016. Development and use of molecular markers: past and present. Crit. Rev. Biotechnol. 36:290-302.

Grünwald, N. J., Garbelotto, M., Goss, E. M., Heungens, K., and Prospero, S. 2012. Emergence of the sudden oak death pathogen Phytophthora ramorum. Trends Microbiol. 20:131-138.

Grünwald, N. J., Goss, E. M., Ivors, K., Garbelotto, M., Martin, F. N., Prospero, S., Hansen, E., Bonants, P. J. M., Hamelin, R. C., Chastagner, G., Werres, S., Rizzo, D. M., Abad, G., Beales, P., Bilodeau, G. J., Blomquist, C. L., Brasier, C., Briere, S. C., Chandelier, A., Davidson, J. M., Denman, S., Elliott, M., Frankel, S. J., Goheen, E. M., de Gruyter, H., Heungens, K., James, D., Kanaskie, A., McWilliams, M. G., Man in 't Veld, W., Moralejo, E., Osterbauer, N. K., Palm, M. E., Parke, J. L., Sierra, A. M. P., Shamoun, S. F., Shishkoff, N., Tooley, P. W., Vettraino, A. M., Webber, J., and Widmer, T. L. 2009. Standardizing the nomenclature for clonal lineages of the sudden oak death pathogen, Phytophthora ramorum. Phytopathology 99:792-795.

Guichoux, E., Lagache, L., Wagner, S., Chaumeil, P., Léger, P., Lepais, O., Lepoittevin, C., Malausa, T., Revardel, E., and Salin, F. 2011. Current trends in microsatellite genotyping. Mol. Ecol. Resour. 11:591-611.

Hurlbert, S. H. 1971. The nonconcept of species diversity: A critique and alternative parameters. Ecology 52:577-586.

Ivors, K. L., Garbelotto, M., Vries, I. D. E., Ruyter-Spira, C., Hekkert, B. T., Rosenzweig, N., and Bonants, P. J. M. 2006. Microsatellite markers identify three lineages of Phytophthora ramorum in US nurseries, yet single lineages in US forest and European nursery populations. Mol. Ecol. 15:1493-1505.

Ivors, K. L., Hayden, K. J., Bonants, P. J. M., Rizzo, D. M., and Garbelotto, M. 2004. AFLP and phylogenetic analyses of North American and European populations of Phytophthora ramorum. Mycol. Res. 108:378-392.

Kamvar, Z. N., Tabima, J. F., and Grünwald, N. J. 2014. Poppr: an R package for genetic analysis of populations with clonal, partially clonal, and/or sexual reproduction. PeerJ 2:e281.

Kasuga, T., Bui, M., Bernhardt, E., Swiecki, T., Aram, K., Cano, L. M., Webber, J., Brasier, C., Press, C. M., Grünwald, N. J., Rizzo, D. M., and Garbelotto, M. 2016. Host-induced aneuploidy and phenotypic diversification in the Sudden Oak Death pathogen Phytophthora ramorum. BMC Genomics 17:385

Koressaar, T., and Remm, M. 2007. Enhancements and modifications of primer design program Primer3. Bioinformatics 23:1289-1291.

Li, H., and Durbin, R. 2009. Fast and accurate short read alignment with BurrowsWheeler Transform. Bioinformatics 25:1754-1760.

Li, H., Ruan, J., and Durbin, R. 2008. Mapping short DNA sequencing reads and calling variants using mapping quality scores. Genome Res. 18:1851-1858.

Mascheretti, S., Croucher, P. J. P., Vettraino, A., Prospero, S., and Garbelotto, M. 2008. Reconstruction of the sudden oak death epidemic in California through microsatellite analysis of the pathogen Phytophthora ramorum. Mol. Ecol. 17:2755-2768

Mata Saez, L., McCracken, A. R., Cooke, L. R., O'Neill, P., Grant, M., and Studholme, D. J. 2015. Draft genome sequences of seven isolates of Phytophthora ramorum EU2 from Northern Ireland. Genom. Data 6:191-192.

Miller, P. M. 1955. V-8 juice agar as a general-purpose medium for fungi and bacteria. Phytopathology 45:461-462.

Mittal, N., and Dubey, A. K. 2009. Microsatellite markers-A new practice of DNA based markers in molecular genetics. Pharmacogn. Rev. 3:235-246.
Möller, E. M., Bahnweg, G., Sandermann, H., and Geiger, H. H. 1992. A simple and efficient protocol for isolation of high molecular weight DNA from filamentous fungi, fruit bodies, and infected plant tissues. Nucleic Acids Res. 20:6115.

Nei, M. 1978. Estimation of average heterozygosity and genetic distance from a small number of individuals. Genetics 89:583-590.

Parra, G., Bradnam, K., and Korf, I. 2007. CEGMA: a pipeline to accurately annotate core genes in eukaryotic genomes. Bioinformatics 23:1061-1067.

Petit, R. J., El Mousadik, A., and Pons, O. 1998. Identifying populations for conservation on the basis of genetic markers. Conserv. Biol. 12:844-855.

Pielou, E. C. 1975. Ecological diversity. Wiley, New York.

Prospero, S., Black, J. A., and Winton, L. M. 2004. Isolation and characterization of microsatellite markers in Phytophthora ramorum, the causal agent of sudden oak death. Mol. Ecol. Notes 4:672-674.

Prospero, S., Hansen, E. M., Grünwald, N. J., and Winton, L. M. 2007. Population dynamics of the sudden oak death pathogen Phytophthora ramorum in Oregon from 2001 to 2004. Mol. Ecol. 16:2958-2973.

Prospero, S., Vercauteren, A., Heungens, K., Belbahri, L., and Rigling, D. 2013. Phytophthora diversity and the population structure of Phytophthora ramorum in Swiss ornamental nurseries. Plant Pathol. 62:1063-1071.

Sambles, C., Schlenzig, A., O’Neill, P., Grant, M., and Studholme, D. J. 2015. Draft genome sequences of Phytophthora kernoviae and Phytophthora ramorum lineage EU2 from Scotland. Genom. Data 6:193-194.

Schmieder, R., and Edwards, R. 2011. Quality control and preprocessing of metagenomic datasets. Bioinformatics 27:863-864.

Schoebel, C. N., Brodbeck, S., Buehler, D., Cornejo, C., Gajurel, J., Hartikainen, H., Keller, D., Leys, M., Říčanová, Š., Segelbacher, G., Werth, S., and Csencsics, D. 2013. Lessons learned from microsatellite development for nonmodel organisms using 454 pyrosequencing. J. Evol. Biol. 26:600-611.

Shimizu, M., Kosaka, N., Shimada, T., Nagahata, T., Iwasaki, H., Nagai, H., Shiba, T., and Emi, M. 2002. Universal fluorescent labeling (UFL) method for automated microsatellite analysis. DNA Res. 9:173-178.

Simpson, E. 1949. Measurements of diversity. Nature 163:688.

Simpson, J. T., Wong, K., Jackman, S. D., Schein, J. E., Jones, S. J. M., and Birol, I. 2009. ABySS: A parallel assembler for short read sequence data. Genome Res. 19:1117-1123.

Stoddart, J. A., and Taylor, J. F. 1988. Genotypic diversity: estimation and prediction in samples. Genetics 118:705-711.

Swain, S., and Garbelotto, M. 2016. Phytophthora ramorum can survive introduction into finished compost. Calif. Agric. 69:237-241.

Untergasser, A., Cutcutache, I., Koressaar, T., Ye, J., Faircloth, B. C., Remm, M. and Rozen, S. G. 2012. Primer3-new capabilities and interfaces. Nucleic Acids Res. 40:e115.

Van Poucke, K., Franceschini, S., Webber, J. F., Vercauteren, A., Turner, J. A., McCracken, A. R., Heungens, K., and Brasier, C. M. 2012. Discovery of a fourth evolutionary lineage of Phytophthora ramorum: EU2. Fungal Biol. 116:1178-1191.

Vercauteren, A., Boutet, X., D'hondt, L., Van Bockstaele, E., Maes, M., Leus, L., Chandelier, A., and Heungens, K. 2011a. Aberrant genome size and instability of Phytophthora ramorum oospore progenies. Fungal Genet. Biol. 48:537-543.

Vercauteren, A., De Dobbelaere, I., Grünwald, N. J., Bonants, P. J. M., Van Bockstaele, E., Maes, M., and Heungens, K. 2010. Clonal expansion of the Belgian Phytophthora ramorum populations based on new microsatellite markers. Mol. Ecol. 19:92-107.

Vercauteren, A., Larsen, M., Goss, E. M., Grünwald, N. J., Maes, M., and Heungens, K. 2011b. Identification of new polymorphic microsatellite markers in the NA1 and NA2 lineages of Phytophthora ramorum. Mycologia 103:1245-1249.

Vercauteren, A., Riedel, M., Maes, M., Werres, S., and Heungens, K. 2013. Survival of Phytophthora ramorum in Rhododendron root balls and in rootless substrates. Plant Pathol. 62:166-176.

Webber, J. F., Mullett, M., and Brasier, C. M. 2010. Dieback and mortality of plantation Japanese larch (Larix kaempferi) associated with infection by Phytophthora ramorum. New Dis. Rep. 22:19.

Werres, S., Wagner, S., Brand, T., Kaminski, K., and Seipp, D. 2007. Survival of Phytophthora ramorum in recirculating irrigation water and subsequent infection of Rhododendron and Viburnum. Plant Dis. 91:1034-1044. 